\title{
Relationship between Red Cell Distribution Width and Microalbuminuria: A Population-Based Study of Multiethnic Representative US Adults
}

\author{
Luis Afonso $^{a, b}$ Sandip K. Zalawadiya ${ }^{b}$ Vikas Veeranna ${ }^{a, b}$ Sidakpal S. Panaich ${ }^{b}$ \\ Ashutosh Niraja, b Sony Jacoba,b \\ a Division of Cardiology, and bepartment of Internal Medicine, Wayne State University, Detroit Medical Center, \\ Detroit, Mich., USA
}

\section{Key Words}

Microalbuminuria $\cdot$ Red cell distribution width .

Cardiovascular events

\begin{abstract}
Introduction: Microalbuminuria (MA), a renal marker of vascular injury, is an independent predictor of cardiovascular (CV) events. Red cell distribution width (RDW), an emerging $\mathrm{CV}$ risk predictor, has not been evaluated for its association with MA. Methods: We evaluated 8,499 participants of the National Health and Nutrition Examination Survey (NHANES) 1999-2006, where RDW was evaluated as a continuous variable and in quartiles $\left(Q_{1} \leq 12.1, Q_{2} 12.2-12.5, Q_{3} 12.6-13\right.$ and $\left.\mathrm{Q}_{4}>13\right)$. Multivariate adjusted logistic regression analysis was performed to estimate the odds of having MA $(n=1,736$; adjusted for traditional CV risk factors, race, BMI, estimated glomerular filtration rate, hemoglobin, mean corpuscular volume, high-sensitivity C-reactive protein and nutritional factors deficiencies of iron, folate and vitamin $\left.\mathrm{B}_{12}\right)$. Results: The prevalence of MA increased with increasing RDW $(13.52 \%$ in $Q_{1}$ vs. $30.02 \%$ in $\left.Q_{4}, p<0.001\right)$. The odds of having $M A$ for those in $\mathrm{Q}_{4}$ was 2.49 (95\% Cl: 1.95-3.18, $\left.\mathrm{p}<0.001\right)$ compared to those in $\mathrm{Q}_{1}$ after the adjustments. No effect modification was observed by covariates on the association between RDW and MA. Conclusion: Elevated RDW is independently associated with a higher risk of MA. An interaction between
\end{abstract}

\section{KARGER}

Fax +41613061234 E-Mail karger@karger.ch www.karger.com

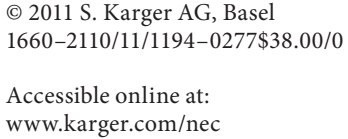

chronic inflammation, oxidative stress, neurohumoral overactivity and endothelial dysfunction may explain this association and the attendant elevated $\mathrm{CV} /$ renal risk.

Copyright $\odot 2011$ S. Karger AG, Basel

\section{Introduction}

Red cell distribution width (RDW), a measure of anisocytosis, has traditionally been used for differential diagnosis of anemia [1], with more recent interest as a marker of higher all-cause and cardiovascular (CV) mortality and morbidity [2-8]. The most plausible explanations put forth for this purported association are chronic inflammation and oxidative stress, leading to elevation in RDW [9-12]. Likewise, an increasing body of evidence suggests that microalbuminuria (MA), a renal marker of vascular damage, is also a reliable predictor of CV risk [13-15] and mortality [16, 17]. Again, the association with chronic subclinical inflammation [18], oxidative stress [19] and endothelial dysfunction [20] are thought to be proximate mediating mechanisms. We hypothesized that a common interaction of the aforementioned pathophysiological processes might link RDW with MA and potentially offer further insight into the observed utility of these biomarkers in ascertaining CV risk. Therefore, in this cross-sectional analysis of the Na- 
tional Health and Nutrition Examination Survey (NHANES) data from 1999 to 2006, we sought to explore the relationship between RDW and MA.

\section{Methods}

\section{Study Population}

NHANES is intended to assess the health and nutritional status of noninstitutionalized nationally representative children and adults in the US. Participants underwent standardized interviews, physical examination and laboratory testing. The study protocols for NHANES 1999-2006 were approved by the National Center for Health Statistics ethics/institutional review board and each participant gave their signed informed consent (for more information see http://www.cdc.gov/nchs/nhanes/irba98.htm).

\section{Data Collection}

Out of a total 41,474 participants, we identified 8,499 individuals applying exclusion criteria like age $<20$ years $(n=21,162)$, evidence of macroalbuminuria $(\mathrm{n}=8,282)$ and individuals with missing information/variables $(n=3,531)$. Data were collected for demographic variables like age, gender and ethnicity (self-reported); medical comorbidities such as hypertension (defined as blood pressure $\geq 140 / 90 \mathrm{~mm} \mathrm{Hg}$ or use of antihypertensive medications or physician diagnosis of hypertension), diabetes mellitus [defined as physician diagnosis of diabetes, taking oral hypoglycemic or insulin for diabetes, nonfasting plasma glucose $\geq 11.1 \mathrm{mmol} / \mathrm{l}(200 \mathrm{mg} /$ dl) or fasting plasma glucose $\geq 7 \mathrm{mmol} / \mathrm{l}$ (126 mg/dl), or hemoglobin $A_{1 c} \geq 6.5 \%$ ], smoking (lifetime smoking of $>100$ cigarettes) and hyperlipidemia (diagnosed by a physician or a serum total cholesterol $\geq 240 \mathrm{mg} / \mathrm{dl}$ or use of lipid lowering therapy). BMI of $<25$ was defined as normal, 25-29.9 as overweight and $\geq 30$ as obese.

\section{Laboratory Measurements}

For detailed description of laboratory measurements, please refer to our prior published work [8]. Briefly, RDW was measured using the Beckman automated Coulter counter method of counting and sizing. Serum creatinine was standardized [1.013·serum creatinine $(\mathrm{mg} / \mathrm{dl})+0.147]$ and estimated glomerular filtration rate (eGFR) was measured using the Modification of Diet in Renal Disease study equation [21]. Individuals were then divided into three categories based on eGFR: $>90$ (reference), 60-90 and $<60 \mathrm{ml} /$ $\min / 1.73 \mathrm{~m}^{2}$. Iron, folate and vitamin $B_{12}$ deficiency were defined per our prior published data [8]. Urine creatinine was analyzed by Jaffe reaction, and urinary albumin was measured using a solidphase fluorescent immunoassay. The urine albumin-creatinine ratio (in $\mathrm{mg} / \mathrm{g}$ ) was calculated by dividing the urinary albumin value by the urinary creatinine concentration. MA was defined as a urine albumin-creatinine ratio $\geq 30 \mathrm{mg} / \mathrm{g}$ and $<300 \mathrm{mg} / \mathrm{g}$. Participants with macroalbuminuria were excluded. Detailed data on NHANES survey operations are available on the NHANES website: http:// www.cdc.gov/nchs/nhanes/nhanes_questionnaires.htm.

\section{Statistical Analysis}

RDW quartiles were generated for the overall population based on its distribution in non-cases (individuals without MA). Baseline characteristics were then compared across these quartiles using a $\chi^{2}$ test for categorical variables and ANOVA test for continuous variables. High-sensitivity C-reactive protein (hsCRP) was log-transformed to normalize its distribution. Spearman's correlation analysis between RDW and the urinary albumin-creatinine ratio (log-transformed) was performed. Multivariate adjusted logistic regression analysis was performed generating different models adjusting for demographics, comorbidities, eGFR, hsCRP, blood hemoglobin, MCV and nutritional factor deficiency (serum iron, folate and vitamin $\mathrm{B}_{12}$ ). The variables were added to each model based on their clinical and statistical significance $(\mathrm{p}<0.20)$ upon univariate logistic regression analysis. Formal analysis for checking interaction between RDW and covariates was also performed. Statistical significance was defined as a twosided p value below 0.05 for the entire analyses. Eight-year sample weights provided by the National Center for Health Statistics were used in all analyses to incorporate the complex survey design of NHANES with appropriate reflections of stratification and clustering. All the analyses were performed using statistical software STATA, version 10 (StataCorp LP, College Station, Tex., USA).

\section{Results}

Table 1 shows baseline characteristic distribution across the quartiles of RDW. Mean age, systolic blood pressure, hemoglobin $A_{1 c}$, serum creatinine and hsCRP along with the proportion of African Americans, coronary artery disease, diabetes, myocardial infarction, stroke, angina and nutritional factor deficiency increased, whereas, mean MCV and eGFR decreased with increasing RDW ( $\mathrm{p}$ for trend $<0.001$ ). Spearman's correlation analysis between RDW and the urinary albumin-creatinine ratio showed a significant coefficient of 0.16 ( $p<$ 0.001; fig. 1).

As shown in table 2, a graded increase in proportion of individuals with MA with increasing quartiles of RDW (13.52\% in $\mathrm{Q}_{1}$ vs. $30.02 \%$ in $\mathrm{Q}_{4}, \mathrm{p}<0.001$ ) was observed. RDW as a continuous variable [adjusted OR (aOR): 1.34, 95\% CI: $1.23-1.46, \mathrm{p}<0.001)$ and a categorical variable (aOR: 2.49 , 95\% CI: 1.95-3.18, $\mathrm{p}<0.001$ ) remained a statistically significant predictor of MA in model 5 (the most adjusted model). The trend of increasing odds of MA with increasing quartiles of RDW persisted across the range of adjustments as shown in table 2 ( $\mathrm{p}$ for the trend $<0.001$ ).

The interaction terms between RDW and various independent variables like gender $(p=0.513)$, ethnicity $(\mathrm{p}=0.081)$, BMI $(\mathrm{p}=0.842)$, hypertension $(\mathrm{p}=0.068)$, hyperlipidemia $(\mathrm{p}=0.788)$, diabetes $(\mathrm{p}=0.399)$ and eGFR $(\mathrm{p}=0.117)$ were found to be nonsignificant in model 5 . Furthermore, we found that the aOR for RDW was 1.56 (model 5; 95\% CI: 1.01-1.39, p < 0.05) when hsCRP levels were $\geq 1 \mathrm{mg} / \mathrm{l}$ and 1.31 (model 5; 95\% CI: 1.13-1.51, $\mathrm{p}<$ 0.05 ) when hsCRP levels were below $1 \mathrm{mg} / \mathrm{l}$ ( $\mathrm{p}$ for interaction 0.188). 
Table 1. Baseline characteristics across RDW quartiles $(\mathrm{n}=8,499)$

\begin{tabular}{|c|c|c|c|c|c|}
\hline & $\begin{array}{l}\mathrm{Q}_{1} \\
(\leq 12.1 \\
\mathrm{n}=1,827)\end{array}$ & $\begin{array}{l}\mathrm{Q}_{2} \\
(12.2-12.5 \\
\mathrm{n}=2,591)\end{array}$ & $\begin{array}{l}\mathrm{Q}_{3} \\
(12.6-13 \\
\mathrm{n}=1,709)\end{array}$ & $\begin{array}{l}\mathrm{Q}_{4} \\
(>13 \\
\mathrm{n}=2,372)\end{array}$ & $\begin{array}{l}\mathrm{p} \\
\text { value }\end{array}$ \\
\hline Age, years & $46.0 \pm 18.5$ & $48.9 \pm 18.7$ & $52 \pm 19$ & $54 \pm 20$ & $<0.001$ \\
\hline Sex (male) & 44.9 & 49.4 & 48.1 & 46.5 & 0.005 \\
\hline \multicolumn{6}{|l|}{ Race, \% } \\
\hline African American & 15.5 & 17.8 & 20.3 & 26.3 & \multirow[t]{4}{*}{$<0.001$} \\
\hline Hispanic American & 29.7 & 29.5 & 29.4 & 26.6 & \\
\hline Caucasian American & 50.8 & 49.4 & 46.6 & 43.4 & \\
\hline Other American & 4.1 & 3.3 & 3.7 & 3.7 & \\
\hline History of CAD & 3.3 & 4.4 & 4.4 & 6.3 & $<0.001$ \\
\hline History of diabetes & 11.9 & 13.7 & 15.2 & 17.6 & $<0.001$ \\
\hline History of myocardial infarction & 3.4 & 4.3 & 4.1 & 7.7 & $<0.001$ \\
\hline History of stroke & 2.4 & 3.4 & 4.00 & 5.6 & $<0.001$ \\
\hline History of angina & 2.7 & 3.1 & 4.5 & 4.7 & $<0.001$ \\
\hline History smoking ( $>100$ cigarettes in lifetime) & 45.8 & 47.3 & 49.4 & 49.1 & 0.091 \\
\hline Family history of CAD & 12.9 & 12.0 & 11.6 & 11.9 & 0.696 \\
\hline Systolic blood pressure, $\mathrm{mm} \mathrm{Hg}$ & $124 \pm 19$ & $126 \pm 20$ & $129 \pm 22$ & $130 \pm 23$ & \\
\hline Antihypertension medication use & 17.9 & 21.6 & 27.2 & 30.6 & $<0.001$ \\
\hline \multicolumn{6}{|l|}{ BMI } \\
\hline Overweight & 37.6 & 38.6 & 34.8 & 34.4 & \multirow[t]{2}{*}{$<0.001$} \\
\hline Obese & 27.9 & 32.1 & 36.1 & 37.7 & \\
\hline Blood hemoglobin, g \% & $14.3 \pm 1.5$ & $14.4 \pm 1.5$ & $14.3 \pm 1.5$ & $13.8 \pm 1.7$ & $<0.001$ \\
\hline Mean corpuscular volume, mean fl & $91.0 \pm 5.0$ & $90.6 \pm 4.8$ & $90.5 \pm 4.9$ & $88.7 \pm 6.8$ & $<0.001$ \\
\hline Total cholesterol, mg \% & $201 \pm 44$ & $203 \pm 43$ & $204 \pm 40$ & $202 \pm 42$ & 0.133 \\
\hline Serum high-density lipoprotein, mg \% & $52 \pm 16$ & $53 \pm 15$ & $53 \pm 16$ & $53 \pm 16$ & 0.407 \\
\hline Hemoglobin $\mathrm{A}_{1 \mathrm{c}}, \%$ & $5.5 \pm 1.3$ & $5.6 \pm 1.1$ & $5.7 \pm 1.1$ & $5.7 \pm 1.2$ & $<0.001$ \\
\hline Standardized serum creatinine, $\mathrm{mg} / \mathrm{dl}$ & $0.95 \pm 0.33$ & $0.99 \pm 0.44$ & $1.0 \pm 0.34$ & $1.1 \pm 0.58$ & $<0.001$ \\
\hline $\mathrm{eGFR}, \mathrm{ml} / \mathrm{min} / 1.73 \mathrm{~m}^{2}$ & $82.5 \pm 23.6$ & $80.5 \pm 24.9$ & $78.4 \pm 27.0$ & $76.7 \pm 27.6$ & $<0.001$ \\
\hline Serum CRP, mg/l & $0.44 \pm 0.90$ & $0.45 \pm 0.75$ & $0.53 \pm 0.97$ & $0.68 \pm 1.28$ & $<0.001$ \\
\hline MA & 13.5 & 16.5 & 20.5 & 30.0 & $<0.001$ \\
\hline Deficiency of nutritional factors ${ }^{a}$ & 4.1 & 6.0 & 8.0 & 23.2 & $<0.001$ \\
\hline Iron deficiency & 2.5 & 5.0 & 7.1 & 26.4 & $<0.001$ \\
\hline Folate deficiency & 0.35 & 0.2 & 0.3 & 0.9 & 0.006 \\
\hline Vitamin $\mathrm{B}_{12}$ deficiency & 1.9 & 2.4 & 2.5 & 3.9 & 0.001 \\
\hline
\end{tabular}

Values are either means $\pm \mathrm{SD}$ or percentages. $\mathrm{CAD}=$ Coronary artery disease.

a Data available for 7,603 participants only.

Upon sensitivity analyses applying adjustments per model 5 (data not shown), the association between RDW and MA persisted after excluding participants with RDW >15\% (aOR: 1.55, 95\% CI: 1.38-1.74, p < 0.001), prior history of CVD (history of coronary artery disease, stroke and congestive heart failure; aOR: 1.50, 95\% CI: $1.32-1.71, \mathrm{p}<0.001)$, diabetes (aOR: 1.56, 95\% CI: $1.35-1.80, \mathrm{p}<0.001$ ), deficiency of nutritional factors (aOR: 1.63, 95\% CI: 1.42-1.87, p < 0.001) and anemics (defined as blood hemoglobin below $13 \mathrm{~g} / \mathrm{dl}$ for males and below $12 \mathrm{~g} / \mathrm{dl}$ for females; aOR: 1.59, 95\% CI: 1.38 $1.83, \mathrm{p}<0.001)$.

Relationship between RDW and MA

\section{Discussion}

Our study results provide novel insight into the association between RDW and MA. First, we observed that RDW increases significantly as the degree of the urinary albumin-creatinine ratio (mg/g) increases. Second, a graded association between RDW and MA was observed, with the risk of MA increasing as RDW values increase. Third, the relationship between RDW and MA maintained significance across gender and ethnicity strata, independent of potential confounder comorbidities (i.e. hypertension, diabetes and obesity). Fourth, although a

Nephron Clin Pract 2011;119:c277-c282 
Fig. 1. Spearman's correlation between RDW and the urinary albumin-creatinine ratio (mg/g; log-transformed). Analysis excludes extreme observations.

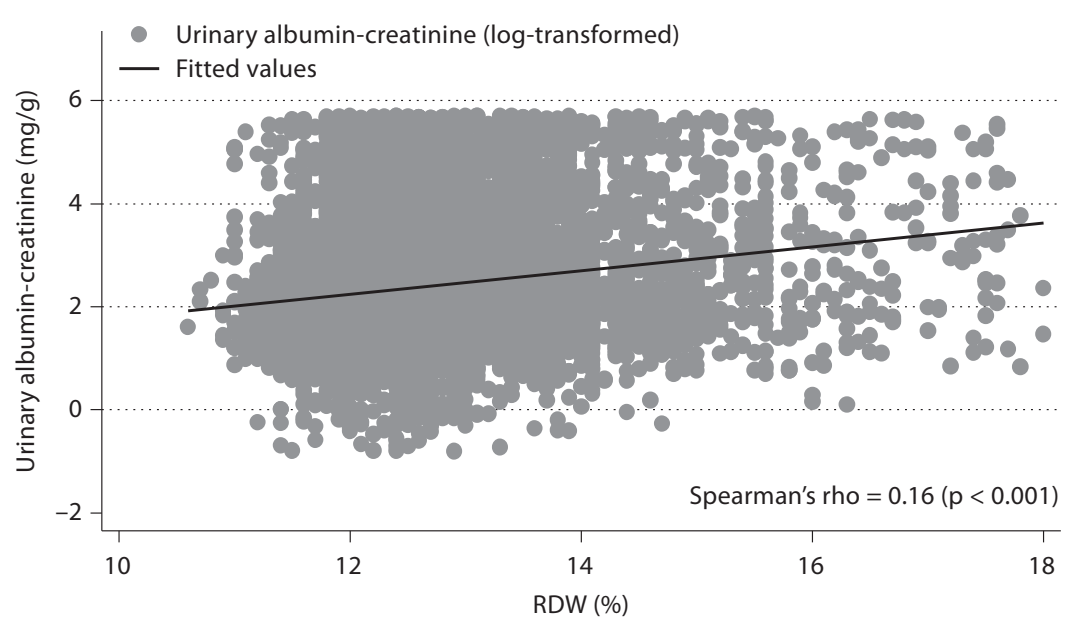

Table 2. RDW and overall odds of having microalbuminuria

a RDW as a continuous variable

\begin{tabular}{llll}
\hline & OR & $95 \%$ CI & p value \\
\hline Model 1 & 1.33 & $1.24-1.42$ & $<0.001$ \\
Model 2 & 1.33 & $1.24-1.42$ & $<0.001$ \\
Model 3 & 1.32 & $1.23-1.41$ & $<0.001$ \\
Model 4 & 1.34 & $1.24-1.45$ & $<0.001$ \\
Model 5 & 1.34 & $1.23-1.46$ & $<0.001$ \\
\hline
\end{tabular}

b RDW quartiles

\begin{tabular}{|c|c|c|c|c|c|c|c|c|c|c|}
\hline \multirow[t]{2}{*}{ Cases: } & \multirow[t]{2}{*}{$\begin{array}{l}\mathrm{Q}_{1}(\mathrm{n}=1,827) \\
247(13.52 \%)\end{array}$} & \multicolumn{3}{|c|}{$\begin{array}{l}\mathrm{Q}_{2}(\mathrm{n}=2,591) \\
427(16.48 \%)\end{array}$} & \multicolumn{3}{|c|}{$\begin{array}{l}\mathrm{Q}_{3}(\mathrm{n}=1,709) \\
350(20.48 \%)\end{array}$} & \multicolumn{3}{|c|}{$\begin{array}{l}\mathrm{Q}_{4}(\mathrm{n}=2,372) \\
712(30.02 \%)\end{array}$} \\
\hline & & OR & $95 \% \mathrm{CI}$ & $\mathrm{p}$ value & OR & $95 \% \mathrm{CI}$ & $\mathrm{p}$ value & OR & $95 \%$ CI & $\mathrm{p}$ value \\
\hline Model 1 & reference & 1.15 & $0.90-1.48$ & 0.257 & 1.61 & $1.28-2.02$ & $<0.001$ & 2.56 & $2.06-3.18$ & $<0.001$ \\
\hline Model 2 & reference & 1.14 & $0.88-1.46$ & 0.270 & 1.61 & $1.27-2.04$ & $<0.001$ & 2.55 & $2.05-3.16$ & $<0.001$ \\
\hline Model 3 & reference & 1.14 & $0.89-1.45$ & 0.285 & 1.64 & $1.30-2.06$ & $<0.001$ & 2.51 & $2.03-3.10$ & $<0.001$ \\
\hline Model 4 & reference & 1.17 & $0.91-1.50$ & 0.223 & 1.64 & $1.30-2.08$ & $<0.001$ & 2.45 & $1.95-3.08$ & $<0.001$ \\
\hline Model $5^{*}$ & reference & 1.17 & $0.89-1.54$ & 0.247 & 1.69 & $1.31-2.18$ & $<0.001$ & 2.49 & $1.95-3.18$ & $<0.001$ \\
\hline
\end{tabular}

Model 1: RDW, adjusted for age, sex and race; model 2: age, sex, race, BMI, hypertension, hyperlipidemia, history of smoking, diabetes; model 3: model $2+$ eGFR and hsCRP; model 4: model $3+$ hemoglobin and mean corpuscular volume; model 5: model $4+$ deficiency of nutritional factors (iron, folate and vitamin $\left.\mathrm{B}_{12}\right) .{ }^{*}$ Analysis done for individuals after excluding individuals with missing data $(\mathrm{n}=$ 7,603).

graded increase in the strength of association between RDW and MA with increasing degrees of inflammation (ascertained by hsCRP levels) was observed, this relationship retained significance in the setting of normal hsCRP levels as well (hsCRP $<1 \mathrm{mg} / \mathrm{l}$ ).
An attempt to understand the underlying pathophysiological association between RDW and MA might shed further light into the interaction and pathobiology of these nontraditional biomarkers as CV risk prognosticators. RDW has been proposed as a marker of chronic in- 


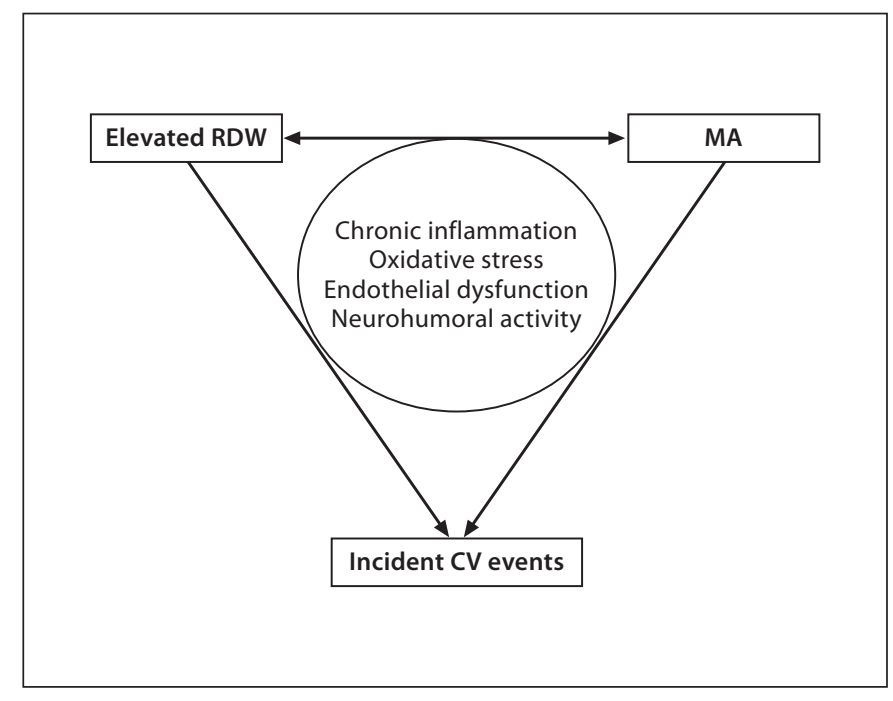

Fig. 2. Pathophysiological interplay between RDW and MA as predictors of $\mathrm{CV}$ events.

flammation $[10,12]$. Similarly, MA has also been associated with the chronic inflammatory state [18] and has been implicated in the origin of early kidney damage [18]. Chronic inflammation has been shown to promote abnormal mesangial cell proliferation and increased vascular permeability leading to albuminuria [22]. Thus, albuminuria appears to reflect a higher level of chronic inflammation, which provides a plausible explanation for its association with higher RDW and adverse CV events.

In the present study, we observed a consistent relationship between RDW and MA across hsCRP strata. Significant association between RDW and MA at normal hsCRP levels suggests that clinically relevant increases in RDW levels may be present in individuals with a normal hsCRP level. Along these lines, while inflammation has been shown to lead to anisocytosis, the association between RDW and mortality has been noted to be independent of hsCRP levels [7]. It is tempting to speculate that higher RDW may well be an earlier marker of chronic subclinical inflammation than hsCRP. Alternatively, pathophysiological processes which elevate RDW other than chronic inflammation (e.g. higher oxidative stress, endothelial dysfunction and neurohumoral overactivity) might be responsible for the association between RDW and MA at normal hsCRP levels.

RDW is also found to be elevated in conditions associated with higher oxidative stress $[23,24]$ and has been shown to be associated with markers of oxidative stress
[10]. Increased oxidative stress can induce glomerular disruption and MA $[19,22]$, which in turn might explain the association with elevated RDW values.

Overstimulation of neurohumoral activity has been shown to suppress erythropoiesis and induce anemia independent of serum erythropoietin levels [25]. Prior research of patients with congestive heart failure, a condition with heightened neurohumoral activity [26], has shown that RDW levels are chronically elevated in these patients and are associated with adverse outcomes including CV morbidity and mortality [2-4]. Similarly, higher levels of neurohumoral activity have been independently associated with a higher incidence of MA [27]. Therefore, a common ground of sympathetic overactivity might also explain the association between elevated RDW and MA.

Our study findings indicate a graded association between RDW and MA. Although the precise pathophysiologic link between this interaction was not examined in the present study, common interactions between inflammation, endothelial dysfunction, oxidative stress and neurohumoral overactivity may explain the observed association and the emerging utility of these biomarkers in the prediction of $\mathrm{CV}$ events (fig. 2).

Our study is a cross-sectional analysis with its inherent limitations and is thus unable to definitively comment on the causality/temporal association. Laboratory values reported in the NHANES dataset are single occasion measurements and it is possible that serial evaluations might allow superior characterization of the association between RDW and MA. However, our study derives strength from a multiethnic community-based sample cohort representative of the national US population with comprehensive evaluation that allows adjustment for multiple confounding factors.

In conclusion, our observations reveal a graded positive relationship between RDW and MA independent of multiple confounders including hsCRP. A complex interplay of overlapping pathophysiological processes might explain this novel association and their increasingly recognized linkage to adverse CV outcomes.

\section{Disclosure Statement}

The contents are solely the responsibility of the authors and do not represent the official view of any organization. The authors have no conflicts of interest or financial disclosures to report.

Nephron Clin Pract 2011;119:c277-c282 c281 


\section{References}

-1 Aslan D, Gumruk F, Gurgey A, Altay C: Importance of RDW value in differential diagnosis of hypochrome anemias. Am J Hematol 2002;69:31-33.

$\checkmark 2$ Felker GM, Allen LA, Pocock SJ, et al: Red cell distribution width as a novel prognostic marker in heart failure: data from the CHARM Program and the Duke Databank. J Am Coll Cardiol 2007;50:40-47.

$\checkmark 3$ van Kimmenade RR, Mohammed AA, Uthamalingam S, van der Meer P, Felker GM, Januzzi JL Jr: Red blood cell distribution width and 1-year mortality in acute heart failure. Eur J Heart Fail 2010;12:129-136.

$\checkmark 4$ Zalawadiya SK, Zmily H, Farah J, Daifallah S, Ali O, Ghali JK: Red cell distribution width and mortality in predominantly African-American population with decompensated heart failure. J Card Fail 2011;17:292298.

5 Patel KV, Ferrucci L, Ershler WB, Longo DL, Guralnik JM: Red blood cell distribution width and the risk of death in middle-aged and older adults. Arch Intern Med 2009;169: 515-523.

6 Patel KV, Semba RD, Ferrucci L, et al: Red cell distribution width and mortality in older adults: a meta-analysis. J Gerontol A Biol Sci Med Sci 2010;65:258-265.

$\checkmark 7$ Perlstein TS, Weuve J, Pfeffer MA, Beckman JA: Red blood cell distribution width and mortality risk in a community-based prospective cohort. Arch Intern Med 2009;169: 588-594.

$>8$ Zalawadiya SK, Veeranna V, Niraj A, Pradhan J, Afonso L: Red cell distribution width and risk of coronary heart disease events. Am J Cardiol 2010;106:988-993.

>9 Forhecz Z, Gombos T, Borgulya G, Pozsonyi Z, Prohaszka Z, Janoskuti L: Red cell distribution width in heart failure: prediction of clinical events and relationship with markers of ineffective erythropoiesis, inflammation, renal function, and nutritional state. Am Heart J 2009;158:659-666.
10 Semba RD, Patel KV, Ferrucci L, et al: Serum antioxidants and inflammation predict red cell distribution width in older women: the Women's Health and Aging Study I. Clin Nutr 2010;29:600-604.

11 Lippi G, Targher G, Montagnana M, Salvagno GL, Zoppini G, Guidi GC: Relation between red blood cell distribution width and inflammatory biomarkers in a large cohort of unselected outpatients. Arch Pathol Lab Med 2009;133:628-632.

12 Allen LA, Felker GM, Mehra MR, et al: Validation and potential mechanisms of red cell distribution width as a prognostic marker in heart failure. J Card Fail 2010;16:230-238.

13 Deveci OS, Kabakci G, Tulumen E, et al: The relationship between microalbuminuria and the presence and extent of coronary atherosclerosis. Angiology 2010;61:184-191.

14 Klausen K, Borch-Johnsen K, Feldt-Rasmussen $B$, et al: Very low levels of microalbuminuria are associated with increased risk of coronary heart disease and death independently of renal function, hypertension, and diabetes. Circulation 2004;110:32-35.

15 Arnlov J, Evans JC, Meigs JB, et al: Lowgrade albuminuria and incidence of cardiovascular disease events in nonhypertensive and nondiabetic individuals: the Framing ham Heart Study. Circulation 2005;112:969975.

16 Gerstein HC, Mann JF, Yi Q, et al: Albuminuria and risk of cardiovascular events, death, and heart failure in diabetic and nondiabetic individuals. JAMA 2001;286:421-426.

17 Weir MR: Microalbuminuria and cardiovascular disease. Clin J Am Soc Nephrol 2007;2: 581-590.
18 Shantha GP, Kumar AA, Bharadhi MK, Arthur P: Role of gender in the associations of microalbuminuria with inflammatory markers in hypertensive subjects: a crosssectional study. Kidney Blood Press Res 2009;32:434-439.

19 Kassab A, Ajmi T, Issaoui M, Chaeib L, Miled A, Hammami M: Homocysteine enhances LDL fatty acid peroxidation, promoting microalbuminuria in type 2 diabetes. Ann Clin Biochem 2008;45:476-480.

20 Lin J, Glynn RJ, Rifai N, et al: Inflammation and progressive nephropathy in type 1 diabetes in the diabetes control and complications trial. Diabetes Care 2008;31:23382343.

21 Levey AS, Coresh J, Greene T, et al: Using standardized serum creatinine values in the Modification of Diet in Renal Disease study equation for estimating glomerular filtration rate. Ann Intern Med 2006;145:247254.

22 Satchell SC, Tooke JE: What is the mechanism of microalbuminuria in diabetes: a role for the glomerular endothelium? Diabetologia 2008;51:714-725.

23 Kiefer CR, Snyder LM: Oxidation and erythrocyte senescence. Curr Opin Hematol 2000; 7:113-116.

24 Garcez ME, Peres W, Salvador M: Oxidative stress and hematologic and biochemical parameters in individuals with Down syndrome. Mayo Clin Proc 2005;80:1607-1611.

25 Fonseca RB, Mohr AM, Wang L, Sifri ZC, Rameshwar P, Livingston DH: The impact of a hypercatecholamine state on erythropoiesis following severe injury and the role of IL6. J Trauma 2005;59:884-889, discussion 889-890.

-26 Francis GS, Goldsmith SR, Levine TB, Olivari MT, Cohn JN: The neurohumoral axis in congestive heart failure. Ann Intern Med 1984;101:370-377.

-27 Takahashi N, Anan F, Nakagawa M, et al: Microalbuminuria, cardiovascular autonomic dysfunction, and insulin resistance in patients with type 2 diabetes mellitus. Metabolism 2004;53:1359-1364. 